\title{
Schwarz Waveform Relaxation Algorithms with Nonlinear Transmission Conditions for Reaction-Diffusion Equations
}

Filipa Caetano ${ }^{1}$, Martin J. Gander ${ }^{2}$, Laurence Halpern ${ }^{3}$ and Jérémie Szeftel ${ }^{4}$

1 Univ. Paris-Sud, Département de Mathématiques; CNRS, F-91405 Orsay, filipa.caetano@math.u-psud.fr

2 Section de Mathématiques, Université de Genève, CP 64, 1211 Genève, Switzerland, martin.gander@math.unige.ch

3 Université Paris 13, CNRS, UMR 7539 LAGA, 99 av. Jean-Baptiste Clément, F-93430 Villetaneuse, France, halpern@math.univ-paris13.fr

4 DMA, Ecole Normale Supérieure, 45 rue d'Ulm, Paris, France, szeftel@dma.ens.fr

\section{Introduction}

Our objective is to develop efficient parallel algorithms for reactive transport equations, which appear in problems related to the numerical simulation of geological $\mathrm{CO}_{2}$ storage. We present in this paper a new class of Schwarz waveform relaxation (SWR) algorithms with nonlinear transmission conditions for the model problem of semilinear reaction diffusion equations. These methods are based on the partition of the spatial domain into smaller sub-domains, and then on the approximation of the restriction of the solution to each subdomain. Transmission conditions at the interfaces must be defined in order to couple the problems between sub-domains. In the case of linear advectionreaction-diffusion equations, different types of transmission conditions were considered in Japhet et al. [1998] and in Martin [2005], in dimensions 1 and 2, and partially or numerically optimized. The optimization problem was then solved for Robin transmission conditions in Gander and Halpern [2007], and for higher order conditions in Bennequin et al. [2009]. We are interested here in nonlinear problems and develop for the first time associated non-linear algorithms. After introducing our problem in section 2, we define in section 3 a Schwarz waveform relaxation algorithm together with the different types of transmission conditions that we consider: Robin, second order and nonlinear transmission conditions. The nonlinear conditions are based on best approximation problems for the linearized equation and provide an efficient algorithm. Section 4 is devoted to the numerical implementation of the iterative algorithm and finally, in section 5 , we present numerical results that illustrate the performance of our new algorithms. 


\section{Problem description}

We consider the semilinear reaction diffusion equation in two dimensions

$$
u_{t}-\nu \Delta u+f(u)=0, \text { in } \mathbb{R}^{2} \times(0, T),
$$

with initial condition

$$
u(\cdot, \cdot, 0)=u_{0}(\cdot, \cdot),
$$

where $T>0$ and the diffusion coefficient $\nu$ is a strictly positive constant. Let us suppose that $u_{0} \in H^{2}\left(\mathbb{R}^{2}\right)$ and $f \in \mathcal{C}^{2}(\mathbb{R})$ are given and that $f$ satisfies $f(0)=0$. A weak solution of problem (1)-(2) is defined to be a function $u \in L^{2}\left(0, T ; H^{1}\left(\mathbb{R}^{2}\right)\right) \cap \mathcal{C}\left([0, T] ; L^{2}\left(\mathbb{R}^{2}\right)\right)$, such that $f(u) \in L^{2}\left(0, T ; L^{2}\left(\mathbb{R}^{2}\right)\right)$, satisfying for all $v \in H^{1}\left(\mathbb{R}^{2}\right)$

$$
\frac{d}{d t}(u, v)+\nu(\nabla u, \nabla v)+(f(u), v)=0, \quad \text { in } \mathcal{D}^{\prime}(0, T),
$$

and $u_{\mid t=0}=u_{0}$, where $(\cdot, \cdot)$ denotes the inner product in $L^{2}\left(\mathbb{R}^{2}\right)$.

Let us recall the following result concerning the well-posedness of the Cauchy problem (1)-(2) (for the proof, see for instance Cazenave and Haraux [1998]):

Theorem 1. If $f \in \mathcal{C}^{2}(\mathbb{R})$ and $u_{0} \in H^{2}\left(\mathbb{R}^{2}\right)$, then there exists $T>0$ such that problem (1)-(2) possesses a unique weak solution $u \in L^{2}\left(0, T ; H^{1}\left(\mathbb{R}^{2}\right)\right) \cap$ $\mathcal{C}\left([0, T] ; L^{2}\left(\mathbb{R}^{2}\right)\right)$. We have in addition that $u \in L^{\infty}\left(0, T ; H^{2}\left(\mathbb{R}^{2}\right)\right)$.

\section{The Schwarz waveform relaxation algorithm}

We decompose the domain $\mathbb{R}^{2}$ into two sub-domains $\Omega_{1}=(-\infty, 0) \times \mathbb{R}$ and $\Omega_{2}=(0,+\infty) \times \mathbb{R}$. We denote by $\Gamma:=\{0\} \times \mathbb{R}$ the common boundary of $\Omega_{1}$ and $\Omega_{2}$ and by $n_{1}=(1,0)$ and $n_{2}=(-1,0)$ respectively the unit outward normals to $\Omega_{1}$ and $\Omega_{2}$ at $\Gamma$. We introduce the following non-overlapping Schwarz waveform relaxation algorithm to approximate the solution of problem (1)(2). If after step $k$ of the algorithm the pair $\left(u_{1}^{k}, u_{2}^{k}\right)$ is known, we propose to define $\left(u_{1}^{k+1}, u_{2}^{k+1}\right)$ by solving both problems

$$
\begin{aligned}
\partial_{t} u_{i}^{k+1}-\nu \Delta u_{i}^{k+1}+f\left(u_{i}^{k+1}\right) & =0 & & \text { in } \Omega_{i} \times(0, T), \\
u_{i}^{k+1}(\cdot, \cdot, t=0) & =u_{0 \mid \Omega_{i}} & & \text { in } \Omega_{i} \\
B_{i}\left(u_{i}^{k+1}\right) & =B_{i}\left(u_{j}^{k}\right) & & \text { on } \Gamma \times(0, T),
\end{aligned}
$$

for $i=1, j=2$ and $i=2, j=1$, where $B_{1}$ and $B_{2}$ are differential operators to be defined below. To initialize the algorithm, an initial guess $\left(g_{b 1}^{0}, g_{b 2}^{0}\right)$ must be given: at step 0 of the algorithm we solve then both problems $(3), i=1,2$, with transmission conditions replaced respectively by conditions

$$
B_{1}\left(u_{1}^{0}\right)=g_{b 1}^{0} \quad \text { and } \quad B_{2}\left(u_{2}^{0}\right)=g_{b 2}^{0} .
$$


It is well known that the solution of (1)-(2) as well as its normal derivative must be continuous across $\Gamma$. The issue is then to define algorithms which converge rapidly to the solution of this problem in the global domain. For the linear reaction-diffusion equation, the transparent boundary condition at the boundary $\Gamma$ is obtained through a Fourier transform in time and in transverse direction $y$ (see Gander and Halpern [2007] and Bennequin et al. [2009]). A good approximation of the Fourier symbol can be obtained using Robin or second order, so called Ventcel, transmission conditions.

\subsection{Non-overlapping algorithms of order zero and two}

The non-overlapping Schwarz waveform relaxation algorithm of order zero is obtained by performing a zeroth order polynomial approximation of the Fourier symbol of the transparent boundary condition over $\Gamma$, which leads to Robin transmission conditions defined by

$$
B_{i}(u):=\partial_{n_{i}} u+p u, \quad p>0 .
$$

The non-overlapping Schwarz waveform relaxation algorithm of order 2 is obtained by performing a first order polynomial approximation of the Fourier symbol of the transparent boundary condition over $\Gamma$, which leads to the second order (or Ventcel) transmission conditions

$$
B_{i}(u):=\partial_{n_{i}} u+p u+q\left(\partial_{t} u-\nu \partial_{y}^{2} u\right), \quad p>0, q>0 .
$$

\subsection{Well-posedness and convergence}

For $s>\frac{1}{2}$, we introduce the function spaces $H_{s}^{s}\left(\Omega_{i}\right)=\left\{u \in H^{s}\left(\Omega_{i}\right) \mid u_{\Gamma} \in\right.$ $\left.H^{s}(\Gamma)\right\}$. By using a priori estimates in appropriate spaces and the Gronwall lemma, we can extend the results of Gander and Halpern [2007] and Bennequin et al. [2009] for the linear advection-reaction-diffusion equation to the nonlinear case. We obtain the following theorem concerning the wellposedness of the initial and boundary value problems in the sub-domains, and the convergence of the algorithm.

Theorem 2. Let $g_{b 1}^{0}$ and $g_{b_{2}}^{0}$ in $H^{1}\left(0, T ; L^{2}(\Gamma)\right) \cap L^{\infty}\left(0, T ; H^{\frac{1}{2}}(\Gamma)\right), u_{0} \in$ $H^{2}\left(\mathbb{R}^{2}\right), f \in \mathcal{C}^{2}(\mathbb{R}), p>0$ and $q \geq 0$ be given. Then

1. There exists $T>\bar{T}>0$ such that algorithm (3), initialized with (4), and with the transmission operators defined by (6) (or by (5) if $q=0$ ), defines a unique sequence of iterates $\left(u_{1}^{k}, u_{2}^{k}\right)$ such that

$$
u_{i}^{k} \in\left\{\begin{array}{l}
L^{2}\left(0, \bar{T} ; H_{2}^{2}\left(\Omega_{i}\right)\right) \cap L^{\infty}\left(0, \bar{T} ; H^{2}\left(\Omega_{i}\right)\right) \cap H^{1}\left(0, \bar{T} ; L^{2}\left(\Omega_{i}\right)\right), \text { if } q>0, \\
L^{2}\left(0, \bar{T} ; H^{2}\left(\Omega_{i}\right)\right) \cap L^{\infty}\left(0, \bar{T} ; H^{2}\left(\Omega_{i}\right)\right) \cap H^{1}\left(0, \bar{T} ; L^{2}\left(\Omega_{i}\right)\right), \text { if } q=0 .
\end{array}\right.
$$

Furthermore $u_{i \mid \Gamma}^{k} \in H^{1}\left(0, \bar{T} ; L^{2}(\Gamma)\right)$, and $\partial_{t} u_{i}^{k} \in L^{\infty}\left(0, \bar{T} ; L^{2}\left(\Omega_{i}\right)\right)$.

2. The sequence $\left(u_{1}^{k}, u_{2}^{k}\right)$ converges, as $k \rightarrow \infty$, to $\left(u_{\mid \Omega_{1}}, u_{\mid \Omega_{2}}\right)$. 


\section{Discretization}

We discretize the sub-domain problems by finite elements in space and a finite difference in time, implicit for the linear part and explicit for the nonlinear term. We describe here the numerical method. We are interested in the boundary value problem

$$
\begin{aligned}
u_{t}-\nu \Delta u+f(u) & =0, & & \text { in } \Omega_{i} \times(0, T), \\
u_{\mid t=0} & =u_{0}, & & \text { in } \Omega_{i}, \\
\partial_{n_{i}} u+p u+q\left(\partial_{t} u-\nu \partial_{y}^{2} u\right) & =g, & & \text { on } \Gamma \times(0, T),
\end{aligned}
$$

for a given function $g$ defined on $\Gamma \times(0, T)$. We consider $V_{h}$, a finite dimensional subspace of $H^{1}\left(\Omega_{i}\right)$ of finite $\mathbb{P}_{1}$ elements, and a basis $\Phi_{1}, \ldots, \Phi_{M}$ of $V_{h}, N_{1}, \ldots, N_{M}$ being the mesh points. We search an approximate solution $u_{h}(t)=u_{1}(t) \Phi_{1}+\cdots+u_{M}(t) \Phi_{M}$, which satisfies

$$
\begin{aligned}
\left(u_{h}^{\prime}, \phi_{i}\right)+\nu\left(\nabla u_{h}, \nabla \phi_{i}\right)+\nu p\left(u_{h}, \phi_{i}\right)_{\Gamma}+\nu q\left(u_{h}^{\prime}, \phi_{i}\right)_{\Gamma}+ \\
\nu q\left(\frac{\partial u_{h}}{\partial y}, \frac{\partial \phi_{i}}{\partial y}\right)_{\Gamma}+\left(f\left(u_{h}\right), \phi_{i}\right)=\nu\left(g, \phi_{i}\right)_{\Gamma}, \forall i=1, \ldots, M,
\end{aligned}
$$

where $(\cdot, \cdot)$ is the inner product in $L^{2}\left(\Omega_{i}\right)$, and $(\cdot, \cdot)_{\Gamma}$ is the inner product in $L^{2}(\Gamma)$. We denote by $t^{n}=n \Delta t$ the time grid points, and let $u^{n}:=u_{1}^{n} \Phi_{1}+\cdots+$ $u_{M}^{n} \Phi_{M}$ be the approximate solution at time $t^{n}$. If the approximate numerical solution $U^{n}=\left(u_{1}^{n}, \cdots, u_{M}^{n}\right)$ at time $t^{n}$ is given, the solution $U^{n+1}$ at time $t^{n+1}$ is computed by solving the algebraic system

$$
\begin{aligned}
&\left(\frac{M}{\Delta t}+\nu K+\nu M_{\Gamma} \operatorname{Diag}_{p}+\right.\left.\nu \frac{M_{\Gamma}}{\Delta t} \operatorname{Diag}_{q}+\nu K_{\Gamma} \operatorname{Diag}_{q}\right) U^{n+1}= \\
& \frac{\left(M+\nu M_{\Gamma} \operatorname{Diag}_{q}\right)}{\Delta t} U^{n}-M F\left(U^{n}\right)+\nu M_{\Gamma} G^{n+1},
\end{aligned}
$$

where the mass and stiffness matrices are $M_{i, j}=\left(\phi_{j}, \phi_{i}\right), K_{i, j}=\left(\nabla \phi_{j}, \nabla \phi_{i}\right)$, and on the boundary $M_{\Gamma i, j}=\left(\phi_{j}, \phi_{i}\right)_{\Gamma}, K_{\Gamma i, j}=\left(\partial_{y} \phi_{j}, \partial_{y} \phi_{i}\right)$. $\operatorname{Diag}_{p}$ and $\operatorname{Diag}_{q}$ are the diagonal matrices $\operatorname{diag}(p, \ldots, p)$ and $\operatorname{diag}(q, \ldots, q)$, and we set $F\left(U^{n}\right)=\left(f\left(u_{1}^{n}\right), \ldots, f\left(u_{M}^{n}\right)\right)$ and $G^{n+1}=\left(g\left(N_{1}, t^{n+1}\right), \cdots, g\left(N_{M}, t^{n+1}\right)\right)$.

\subsection{Nonlinear transmission conditions}

The linear Robin and second order transmission conditions defined by the operators (5) and (6) imply a choice of the constants $p$ and $q$. In Gander and Halpern [2007] and Bennequin et al. [2009], the authors established asymptotic formulas (in $\Delta t$ ) for the values of $p$ and $q$ that optimize the convergence factor of the algorithm, in the case of the linear advection-reaction-diffusion equation. The results are based on Fourier transforms in time and in the transversal direction $y$, of the error equations, which are just the homogeneous 
counterpart of the equations for $u_{i}^{k}$ in this linear case. For $\partial_{t} u-\nu \Delta u+b u=0$, where $b$ is a positive constant, explicit formulas for the optimal parameters are given,

$$
p_{o p t}^{R}(\Delta t, b, \nu),
$$

in the case of Robin transmission conditions (see Gander and Halpern [2007]), and

$$
\left(p_{\text {opt }}^{V}, q_{o p t}^{V}\right)(\Delta t, b, \nu)
$$

in the case of second order transmission conditions (see Bennequin et al. [2009]).

Such an explicit analysis seems difficult for a nonlinear equation, since on the one hand the equation satisfied by the errors is not the same, and on the other hand we do not know the Fourier transform of the nonlinear term $f(u)$. However, the equation satisfied by the errors $e_{i}^{k}:=u_{i}^{k}-u$ is

$$
\partial_{t} e_{i}^{k}-\nu \Delta e_{i}^{k}+f\left(u_{i}^{k}\right)-f(u)=0,
$$

and a linearization at the solution $u$ gives

$$
\partial_{t} e_{i}^{k}-\nu \Delta e_{i}^{k}+f^{\prime}(u) e_{i}^{k} \simeq 0 .
$$

This motivates our choice of nonlinear transmission conditions, where we replace $b$ by $f^{\prime}(u)$, in the formulas (8) for Robin, and (9) for second order transmission conditions. Considering nonlinear transmission conditions leads to the discretization of the boundary value problem (7), where in the linear operators (5) and (6), the constants $p$ and $(p, q)$ are replaced by non linear functions $p(u)=p_{o p t}^{R}\left(\Delta t, f^{\prime}(u), \nu\right)$ and $(p, q)(u)=\left(p_{o p t}^{V}, q_{o p t}^{V}\right)\left(\Delta t, f^{\prime}(u), \nu\right)$. In this case the diagonal matrices $\operatorname{Diag}_{p}$ and $\operatorname{Diag}_{q}$ are replaced by the timedependent matrices

$$
\operatorname{Diag}_{p}^{n}=\operatorname{diag}\left(p\left(u_{1}^{n}\right), \ldots, p\left(u_{M}^{n}\right)\right), \quad \operatorname{Diag}_{q}^{n}=\operatorname{diag}\left(q\left(u_{1}^{n}\right), \ldots, q\left(u_{M}^{n}\right)\right) .
$$

\subsection{Implementation of the iterative algorithm}

One step of the iterative Schwarz waveform relaxation algorithm consists in solving both initial boundary value problems in each sub-domain and in defining the new boundary conditions for the next step. We must then discretize the operator $\left(u_{k}^{1}, u_{k}^{2}\right) \longrightarrow\left(B_{1}\left(u_{k}^{2}\right), B_{2}\left(u_{k}^{1}\right)\right)$. To do so, we remark that, if at step $k$ of the algorithm, the transmission conditions are defined by

$$
\partial_{n_{i}} u_{i}^{k}+p\left(u_{i}^{k}\right) u_{i}^{k}+q\left(u_{i}^{k}\right)\left(\partial_{t} u_{i}^{k}-\nu \partial_{y}^{2} u_{i}^{k}\right)=g_{i}^{k},
$$

$i=1,2$, (with the possibility to take into account constant functions $p(u$ ) and $q(u)$ or $q(u)=0$ ), at step $k+1$, the transmission conditions are defined by (10), with

$$
g_{i}^{k+1}=-g_{j}^{k}+2 p\left(u_{j}^{k}\right) u_{j}^{k}+2 q\left(u_{j}^{k}\right)\left(\partial_{t} u_{j}^{k}-\nu \partial_{y}^{2} u_{j}^{k}\right),
$$


with $i=1, j=2$ or $i=2, j=1$. Rewriting the transmission condition in this way has the advantage that no normal derivative has to be computed (cf. Gander et al. [2002] for further details on this kind of technique). We discretize then the boundary condition $g_{i}^{k}$ using the discretizations of the corresponding terms defined in the previous paragraphs.

\section{Numerical results}

In this section, the spatial domain is the square $\Omega=(-1,1) \times(0,2)$, which is decomposed into two sub-domains $\Omega_{1}=(-1,0) \times(0,2)$ and $\Omega_{2}=(0,1) \times(0,2)$. The nonlinear function that we test here is the function $f(u)=10(\exp (u)-1)$. We compare in the next figures the results obtained with the linear and nonlinear Robin and second order transmission conditions described in the previous sections. The figures represent the error between the domain decomposition solution obtained after a fixed number of iterations, and the so-called monodomain solution, which corresponds to the numerical solution computed in the global domain $\Omega$, by using the same numerical method. The boundary conditions at the boundary $\partial \Omega$ are of Dirichlet type. We consider three spatial meshes, corresponding to the values of $h=0.125, h=0.0625$ and $h=0.03125$ and two values for the diffusion coefficient $\nu, \nu=0.1$ and $\nu=1$. The time step $\Delta t$ is such that $\Delta t=h$. The time interval is $[0,1]$.

In Figure 1, we compare, in the case $\nu=0.1$, the results obtained with the nonlinear Robin conditions, the nonlinear second order conditions and the linear Robin conditions where the parameter $p$ corresponds to the optimal parameter for the heat equation. These results validate the use of the nonlinear parameters. The use of nonlinear second order conditions gives better results than both linear and nonlinear Robin conditions: the convergence speed of the algorithm with second order transmission conditions is higher than the convergence speed of the algorithm with Robin transmission conditions. This result was also expected, since the second order conditions correspond to a higher order approximation of the transparent boundary condition on $\Gamma$. We obtained the same qualitative results with other nonlinear functions such as $f(u)=u^{3}, f(u)=u^{5}$ and other functions with a polynomial behavior.

\subsection{A simple model in geological $\mathrm{CO}_{2}$ storage modeling}

We present here a very simple model of a reactive system which can appear in the framework of geological $\mathrm{CO}_{2}$ storage modeling. We consider a reactive chemical system with two types of materials, evolving according to the equation

$$
u_{t}-\nu \Delta u+f(x, y, u)=0 .
$$

The nonlinear function $f$ depends on the space variables, describing a heterogeneous distribution of the materials in the spatial domain. Both materials 

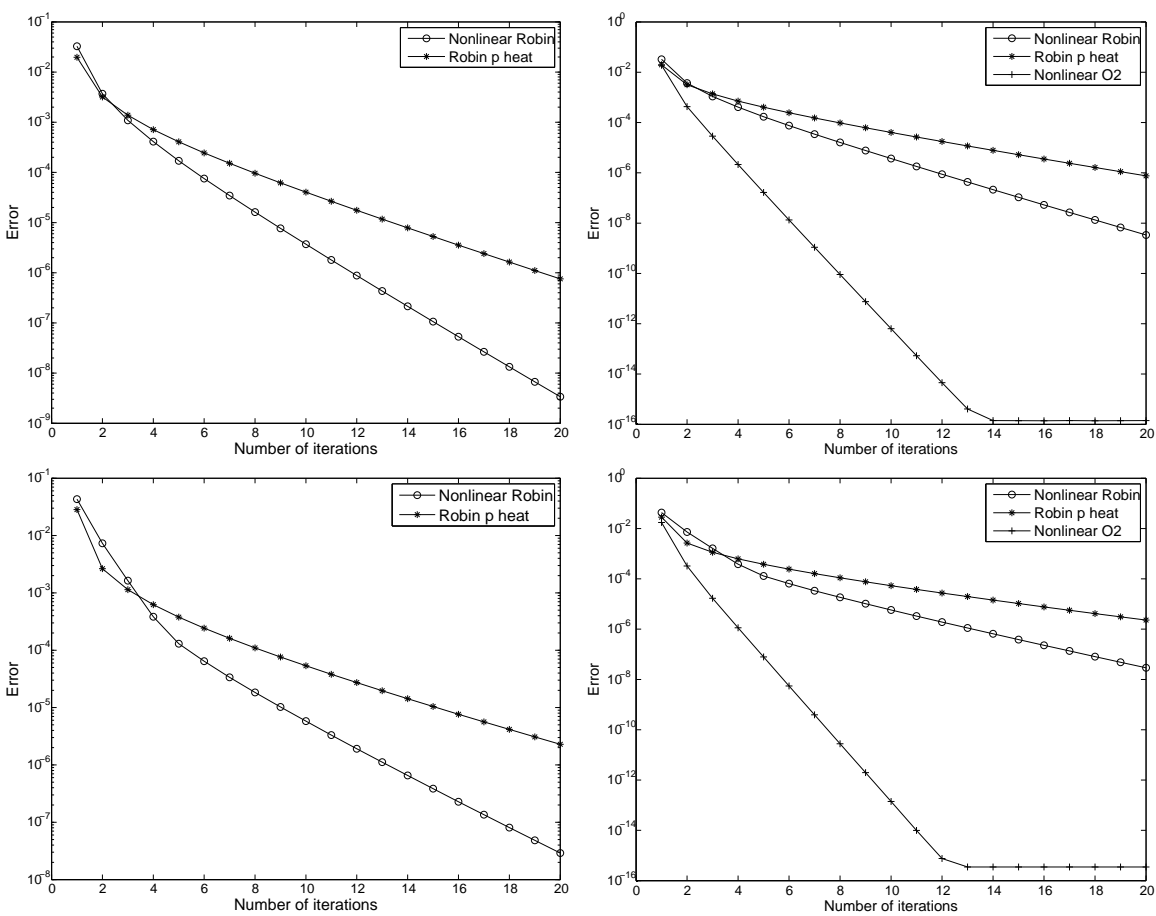

Fig. 1. $f(u)=10(\exp (u)-1), \nu=0.1$ : Difference in the $L^{\infty}\left(0, T ; L^{2}(\Omega)\right)$ norm between domain decomposition and mono-domain solutions, for $h=0.0625$ in the top row, and $h=0.03125$ in the bottom row. On the left, Robin transmission conditions, and on the right Robin and second order transmission conditions

are evolving through equilibrium values $u_{1}^{e q}$ and $u_{2}^{e q}$. The reaction is described here by the function

$$
f(x, y, u)=k_{1} S_{1}(x, y)\left(u-u_{1}^{e q}\right)^{3}+k_{2} S_{2}(x, y)\left(u-u_{2}^{e q}\right)^{3} .
$$

The positive constants $k_{1}$ and $k_{2}$ represent the reaction speeds of material 1 and 2 , and the surface functions $S_{i}$ describe the spatial distribution of the material $i, i=1,2$.

In the test below, we considered $k_{1}=5, u_{1}^{e q}=1, k_{2}=3, u_{2}^{e q}=0$, $S_{1}(x, y)=\sin \left(\frac{3 \pi}{2} x+\frac{\pi}{2}\right) \sin \left(\frac{3 \pi}{2} y+\frac{\pi}{2}\right) \chi_{W}$, where $W$ is a zone corresponding to a part of a circle in the spatial domain, and $S_{2}(x, y)=\max \left(\sin \left(\frac{5 \pi}{2} x+\right.\right.$ $\left.\left.\frac{\pi}{3}\right) \sin \left(\frac{5 \pi}{2} y+\frac{\pi}{3}\right), 0\right)$. The initial and Dirichlet data are both equal to 0.5 . We used here nonlinear Robin and second order transmission conditions, obtained by replacing $b$ with $\partial_{u} f$ in formula (8). In Figure 2 we compare the results obtained with the nonlinear conditions for different values of the mesh-spacing $h$. 

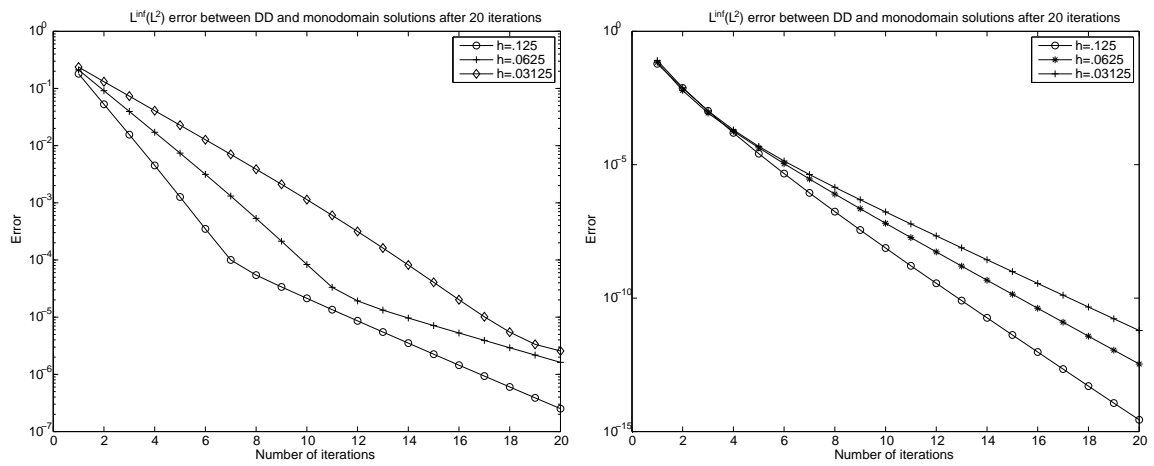

Fig. 2. $f(x, y, u), \nu=1$ : Error after 20 iterations of the algorithm for $h=$ $0.125, h=0.0625$ and $h=0.03125$. On the left, Robin transmission conditions, on the right second order transmission conditions.

Acknowledgments: We thank Anthony Michel for proposing the reactive transport model of the last section, and explaining it to us. This research is supported by the research project SHPCO2 funded by ANR-07-CIS7-007-03.

\section{References}

D. Bennequin, M. J. Gander, and L. Halpern. A homographic best approximation problem with application to optimized Schwarz waveform relaxation. Math. Comp., 78(265):185-223, 2009.

T. Cazenave and A. Haraux. An introduction to semilinear evolution equations, volume 13 of Oxford Lecture Series in Mathematics and its Applications. The Clarendon Press Oxford University Press, New York, 1998.

M. J. Gander and L. Halpern. Optimized Schwarz waveform relaxation methods for advection reaction diffusion problems. SIAM J. Numer. Anal., 45 (2):666-697 (electronic), 2007.

M. J. Gander, F. Magoulès, and F. Nataf. Optimized Schwarz methods without overlap for the Helmholtz equation. SIAM J. Sci. Comput., 24(1):38-60 (electronic), 2002.

C. Japhet, F. Nataf, and F.-X. Roux. Extension of a coarse grid preconditioner to non-symmetric problems. In Domain decomposition methods, 10 (Boulder, CO, 1997), volume 218 of Contemp. Math., pages 279-286. Amer. Math. Soc., Providence, RI, 1998.

V. Martin. An optimized Schwarz waveform relaxation method for the unsteady convection diffusion equation in two dimensions. Appl. Numer. Math., 52(4):401-428, 2005. 